\title{
CONSTITUINTES QUÍMICOS VOLÁTEIS DAS FOLHAS E GALHOS DE Zanthoxylum syncarpum Tull.
}

Mariano George Sousa Vieira, João Vito Barroso de Freitas, Manoel Nelson de Lima Neto e Nilce Viana Gramosa*

Departamento de Química Orgânica e Inorgânica, Centro de Ciências, Universidade Federal do Ceará, CP 6021, 60455-760

Fortaleza - CE, Brasil

Edson Paula Nunes

Departamento de Biologia, Universidade Federal do Ceará, CP 6021, 60455-760 Fortaleza - CE, Brasil

Recebido em 5/5/08; aceito em 22/8/08; publicado na web em 26/1/09

\begin{abstract}
VOLATILE CHEMICAL CONSTITUENTS OF THE LEAVES AND TWIGS FROM Zanthoxylum syncarpum Tull. The essential oils of the leaves and twigs from Zanthoxylum syncarpum Tull. were examined by GC/MS and GC-FID. Variation in the oil composition relative to the harvesting time was also described. The major components in the leaves oils were limonene (23.1-47.3\%) and myrcene (4.8-10.8\%). In the oils of twigs, the main components were ar-curcumene (12.8-18.1\%), $E$ - $\beta$-farnesene (9.1-9.7\%) and $\beta$-caryophyllene (9.2-9.3\%). This paper describes for the first time the composition of the essential oil of the twigs from $Z$. syncarpum.
\end{abstract}

Keywords: Zanthoxylum syncarpum; essential oil composition; Rutaceae.

\section{INTRODUÇÃO}

O gênero Zanthoxylum (Rutaceae) compreende cerca de 200 espécies de árvores, arbustos e lianas com distribuição principalmente pantropical. ${ }^{1}$ Este gênero tem sido objeto de estudo devido principalmente às suas propriedades febrífugas, ${ }^{1-3}$ sudoríferas ${ }^{3,4}$ e diuréticas. ${ }^{3,5}$ Os constituintes majoritários encontrados mais freqüentemente nos óleos essenciais de espécies de Zanthoxylum descritos na literatura são limoneno, ${ }^{6-11}$ germacreno $\mathrm{D},{ }^{2,12-14} \alpha$-pineno ${ }^{14-17}$ e $\beta$-cariofileno..$^{14,18} \mathrm{O}$ limoneno foi encontrado em teores significativos nos óleos das sementes de Z. armatum, ${ }^{12}$ dos frutos de Z. piperitum, ${ }^{6,9}$ Z. limolella ${ }^{8}$ e Z. simulans $^{10}$ e do pericarpo de Z. schinifolium. ${ }^{7}$ Das atividades descritas para óleos essenciais de Zanthoxylum destacam-se: repelente contra o mosquito Aedes aegypti para Z. limonella ${ }^{19}$ e contra insetos para Z. bungenum $^{20}$ e Z. piperitum, ${ }^{21}$ bem como, as atividades larvicida, ${ }^{8}$ inseticida, ${ }^{22,23}$ antimalárica, ${ }^{24}$ antihelmíntica, ${ }^{25}$ ictiotóxica ${ }^{26} \mathrm{e}$ antimicrobiana. ${ }^{4,27}$

No Brasil são descritas 25 espécies de Zanthoxylum, ${ }^{28}$ dentre estas, a espécie Z. syncarpum Tull. conhecida popularmente por limãozinho, ${ }^{3}$ distribuída na região Nordeste, especialmente nos estados do Ceará, Rio Grande do Norte, Paraíba e Pernambuco..$^{29}$ Relatos anteriores referentes aos óleos essenciais desta espécie descrevem os sesquiterpenos $\beta$-elemeno, germacreno $D$ e $\beta$-cariofileno como constituintes majoritários no óleo das folhas, enquanto que no óleo das raízes foram identificados: guai-6(7)-en-11-ol, trans- $\beta$-guaieno, patchouli álcool e guai-7(11)-en-10-ol. ${ }^{3,30}$

O presente trabalho descreve a avaliação dos componentes dos óleos essenciais das folhas e galhos de Z . syncarpum, coletados em duas épocas do ano.

\section{RESULTADOS E DISCUSSÃO}

As folhas de Z. syncarpum coletadas em fevereiro e em julho de 2007 forneceram óleos essenciais com rendimentos de $0,07 \%$ (OEF1) e $0,1 \%$ (OEF2), respectivamente. Foram identificados 25 componentes nestes óleos: 2 álcoois alifáticos, 8 hidrocarbonetos monoterpênicos, 2 monoterpenos oxigenados, 7 hidrocarbonetos sesquiterpênicos e 6 sesquiterpenos oxigenados. Algumas diferenças

*e-mail: nilce@dqoi.ufc.br foram encontradas nas composições dos óleos OEF1 e OEF2 em função do período da coleta. Os álcoois alifáticos foram observados em pequenos teores no óleo de julho $(2,2 \%)$ e ausentes no óleo de fevereiro, enquanto que houve um decréscimo considerável no teor de monoterpenos do óleo coletado em fevereiro $(85,6 \%)$ para o coletado em julho $(54,5 \%)$. O teor dos componentes majoritários de OEF1, limoneno (47,3\%), $\alpha$-felandreno (14,2\%) e mirceno (10,8\%), diminuiu consideravelmente no óleo OEF2 para 23,1\%, ausente e 4,8\%, respectivamente. Já a composição sesquiterpênica de OEF1 teve os teores de $\beta$-elemeno (2,5\%) e espatulenol (ausente) aumentados para 12,6 e $11,4 \%$, respectivamente, em OEF2.

Os galhos coletados em fevereiro e em julho de 2007 forneceram óleos essenciais amarelados com rendimentos bastante semelhantes, compreendendo $0,006 \%$ para OEG1 e $0,005 \%$ para OEG2, respectivamente. Estes óleos não mostraram variações significativas no rendimento e na sua composição em função da época da coleta e, ainda, mostraram ser ricos em sesquiterpenos, diferentemente dos óleos das folhas, ricos em monoterpenos. Dois álcoois alifáticos correspondendo a 2,5\% do total foram identificados somente no óleo coletado em julho, semelhante ao observado para o óleo das folhas. Houve uma pequena variação no teor de sesquiterpenos do óleo obtido em fevereiro $(90,2 \%)$ com relação ao obtido em julho (84,3\%). O constituinte majoritário identificado foi o ar-curcumeno com teores de 12,8 e 18,1\% para OEG1 e OEG2, respectivamente. O germacreno D foi identificado com teor de $9,1 \%$ em OEG1 e não foi identificado em OEG2.

A constituição química dos óleos das folhas OEF1 e OEF2 é coerente com os dados descritos na literatura para óleos essenciais de Zanthoxylum, já que o limoneno é constituinte majoritário em vários óleos deste gênero. ${ }^{6-11}$ De acordo com Petrakis et al., ${ }^{31}$ o limoneno funciona como sinalizador fundamental na interação entre plantas e insetos. Não foram encontrados registros na literatura da presença do ar-curcumeno como constituinte principal.

A variação quantitativa e qualitativa da composição dos óleos das folhas e galhos de Z. syncarpum em estudo pode estar relacionada com uma série de fatores que influenciam sua composição, tais como, fatores ecológicos, estágio de desenvolvimento da planta, sua fisiologia, a idade das folhas, dentre outros. ${ }^{32-34} \mathrm{O}$ fator geográfico não pode ser considerado, já que os óleos estudados foram obtidos do mesmo exemplar da espécie. 
Tabela 1. Composição dos óleos essenciais das folhas e galhos de Z. syncarpum

\begin{tabular}{|c|c|c|c|c|c|c|}
\hline Componentes $^{\mathrm{a}}$ & $\mathrm{IK}-1^{\mathrm{b}}$ & $\mathrm{IK}-2^{\mathrm{c}}$ & OEF1 & OEF2 & OEG1 & OEG2 \\
\hline Álcoois alifáticos & & & - & 2,2 & - & 2,5 \\
\hline Z-3-hexenol & 867 & 859 & - & 1,2 & - & - \\
\hline$E$-2-hexen-1-ol & 869 & ND & - & - & - & 1,3 \\
\hline$n$-hexanol & 877 & 871 & - & 1,0 & - & 1,2 \\
\hline Monoterpenos & & & 85,6 & 54,5 & 4,8 & 6,8 \\
\hline$\alpha$-tujeno & 917 & 930 & 0,7 & - & - & - \\
\hline$\alpha$-pineno & 934 & 939 & 1,4 & 1,9 & - & - \\
\hline sabineno & 970 & 975 & 1,5 & 1,5 & - & - \\
\hline mirceno & 988 & 991 & 10,8 & 4,8 & - & 1,0 \\
\hline$\alpha$-felandreno & 1000 & 1003 & 14,2 & - & - & - \\
\hline$p$-cimeno & 1021 & 1025 & 0,8 & 10,1 & - & - \\
\hline limoneno & 1023 & 1029 & 47,3 & 23,1 & - & - \\
\hline$\beta$-felandreno & 1026 & 1030 & - & - & 2,0 & 2,3 \\
\hline trans- $\beta$-ocimeno & 1044 & 1050 & 6,9 & - & - & 0,7 \\
\hline linalool & 1098 & 1097 & 2,0 & 6,7 & 2,8 & 2,8 \\
\hline criptona & 1183 & 1186 & - & 6,4 & - & - \\
\hline Sesquiterpenos & & & 12,6 & 41,6 & 93,7 & 88,0 \\
\hline$\alpha$-copaeno & 1379 & 1377 & - & 7,0 & 5,4 & 3,9 \\
\hline$\beta$-bourboneno & 1387 & 1388 & - & 1,2 & - & - \\
\hline$\alpha$-duprezianeno & 1395 & 1389 & - & - & 3,5 & 3,0 \\
\hline$\beta$-elemeno & 1398 & 1391 & 2,5 & 12,6 & 5,4 & 3,2 \\
\hline$\alpha$-cis-bergamoteno & 1420 & 1413 & - & - & 4,9 & 4,8 \\
\hline$\beta$-cariofileno & 1425 & 1419 & 0,9 & - & 9,3 & 9,2 \\
\hline$\beta$-duprezianeno & 1435 & 1423 & - & - & - & 0,7 \\
\hline$\alpha$-trans-bergamoteno & 1440 & 1435 & - & - & 6,9 & 6,6 \\
\hline$E$ - $\beta$-farneseno & 1460 & 1457 & - & - & 9,7 & 9,1 \\
\hline sesquisabineno & 1463 & 1460 & - & - & - & 1,1 \\
\hline ar-curcumeno & 1482 & 1481 & - & - & 12,8 & 18,1 \\
\hline germacreno D & 1483 & 1485 & 5,4 & - & 9,1 & - \\
\hline epi-cubebol & 1494 & 1494 & - & 1,7 & - & - \\
\hline biciclogermacreno & 1500 & 1500 & 1,6 & - & 3,6 & 1,2 \\
\hline cubebol & 1515 & 1515 & 0,7 & - & - & - \\
\hline$\beta$-curcumeno & 1516 & 1516 & - & - & 2,1 & 2,3 \\
\hline$\delta$-cadineno & 1523 & 1523 & 0,8 & - & 9,9 & 7,6 \\
\hline hidrato de cis-sesquisabineno & 1548 & 1544 & - & - & - & 1,4 \\
\hline$E$-nerolidol & 1566 & 1563 & - & - & 1,2 & 1,2 \\
\hline espatulenol & 1571 & 1578 & - & 11,4 & 3,9 & 2,4 \\
\hline óxido de cariofileno & 1576 & 1583 & - & 4,3 & 3,2 & 4,4 \\
\hline globulol & 1585 & 1585 & - & - & - & 1,5 \\
\hline viridiflorol & 1592 & 1593 & 0,7 & 1,9 & 1,5 & - \\
\hline 1-epi-cubenol & 1614 & 1629 & - & - & - & 1,9 \\
\hline cubenol & 1627 & 1647 & - & - & - & 1,5 \\
\hline$\alpha$-cadinol & 1636 & 1654 & - & 1,5 & 1,3 & 0,8 \\
\hline$\beta$-bisabolol & 1647 & 1675 & - & - & - & 2,1 \\
\hline Total & - & - & 98,2 & 98,3 & 98,5 & 97,3 \\
\hline
\end{tabular}

${ }^{\mathrm{a} C}$ Componentes listados de acordo com a ordem de eluição em coluna OV-5. b Índices de Kovat corrigidos. ${ }^{\mathrm{c}}$ Índices de Kovat da literatura ${ }^{35}$. ND $=$ Não descrito. 


\section{PARTE EXPERIMENTAL}

\section{Material vegetal}

As folhas e galhos de $Z$. syncarpum foram coletadas de um exemplar desta espécie localizado (coordenadas $03^{\circ} 42^{\prime} 45,4$ " $\mathrm{S}$ e 38³8’30,6” W) no Parque Botânico do Ceará em Caucaia, CE. Foram realizadas coletas das folhas e dos galhos em fevereiro e julho de 2007. O espécime estudado foi identificado pelo Prof. E. P. Nunes, Depto. de Biologia, Universidade Federal do Ceará (UFC) e a exsicata depositada no Herbário Prisco Bezerra-UFC sob número 39.817.

\section{Obtenção dos óleos essenciais}

As folhas e os galhos frescos foram moídos e submetidos ao processo de hidrodestilação utilizando um aparelho tipo Clevenger durante $3 \mathrm{~h}$. Os óleos essenciais obtidos foram acondicionados em frascos de vidro e mantidos sob refrigeração a $-3{ }^{\circ} \mathrm{C}$ até serem analisados. Os óleos obtidos das folhas foram denominados OEF1 e OEF2, referentes às coletas em fevereiro e julho, respectivamente, assim como os óleos dos galhos, denominados OEG1 e OEG2.

\section{Instrumentação e procedimentos experimentais}

Cromatografia gasosa acoplada à espectrometria de massa (CG-EM)

A análise qualitativa do óleo essencial foi realizada utilizando um cromatógrafo gasoso Shimadzu QP5050 equipado com coluna capilar de sílica fundida OV-5 (30 m; 0,25 mm; 0,25 $\mu \mathrm{m})$ acoplado a espectrômetro de massa. $\mathrm{O}$ espectro de massa foi obtido com a voltagem de ionização de $70 \mathrm{eV}$ (temperatura da fonte $250{ }^{\circ} \mathrm{C}$ ). A rampa de temperatura foi programada de $40-180{ }^{\circ} \mathrm{C}$ a $4^{\circ} \mathrm{C} / \mathrm{min}$ e de $180-280{ }^{\circ} \mathrm{C}$ a $20^{\circ} \mathrm{C} / \mathrm{min}$ e mantido um isoterma por $7 \mathrm{~min}$. O gás de arraste utilizado foi o hélio com um fluxo de $1,0 \mathrm{~mL} / \mathrm{min}$.

Cromatografia gasosa com detector de ionização de chama (CG-DIC)

A análise quantitativa foi realizada em cromatógrafo gasoso FID Trace GC Ultra (Thermo Electron Corporation), equipado com uma coluna capilar OV-5 (30 m; 0,25 mm; 0,25 $\mu \mathrm{m}$ ), operando sob as mesmas condições descritas para o CG-EM, e um detector de ionização de chama. Os dados quantitativos foram obtidos das percentagens das áreas do cromatograma do CG-DIC sem o uso de fatores de correção.

\section{Identificação dos constituintes químicos dos óleos essenciais}

Os constituintes foram identificados por comparação de seus espectros de massa com aqueles do banco de dados WILEY229 e confirmados por seus índices de Kovat, que foram calculados em relação aos tempos de retenção de uma série homóloga de n-alcanos. ${ }^{35}$

\section{AGRADECIMENTOS}

À FUNCAP/CNPq/PPP, CNPq, FINEP, PRONEX, PADCT e CAPES pelo apoio financeiro e pelas bolsas concedidas.

\section{REFERÊNCIAS}

1. Rout, P. K.; Naik, S. N.; Rao, Y. R.; Jadeja, G.; Maheshwari, R. C.; J. Supercrit. Fluids 1990, 42, 334.

2. Moura, N. F.; Strapazzon, J. O.; Loro, F.; J. Essent. Oil Res. 2006, 18, 4.

3. Morais, S. M.; Facundo, V. A.; Braz-Filho, R.; J. Essent. Oil Res. 2002, 14, 274.

4. Setzer, W. N.; Schmidt, J. M.; Eiter, L. C.; Haber, W. A.; J. Essent. Oil
Res. 2005, 17, 333

5. Ngane, A. N.; Biyiti L.; Zollo, P. H. A.; Bouchet, P.; J. Ethnopharmacol. 2000, 70, 335 .

6. Choochote, W.; Chaithong, U.; Kamsuk, K.; Jitpakdi, A.; Tippawangkosol, P.; Tuetun, B.; Champakaew, D.; Pitasawat, B.; Fitoterapia 2007, $78,359$.

7. Iseli, V.; Potterat, O.; Hagmann, L.; Egli, J.; Hamburger, M.; Pharmazie 2007, 62, 396.

8. Pitasawat, B.; Champakew, D.; Choochote, W.; Jitpakdi, A.; Chaithong, U.; Kanjanapothi, D.; Rattanachanpichai, E.; Tippawangkosol, P.; Riyong, D.; Tuetun, B.; Chaiyasit, D.; Fitoterapia 2007, 78, 205.

9. Lihua, J.; Kikue, K.; J. Agric. Food Chem. 2004, 52, 4197.

10. Charng-Cherng, C.; Jeng-Leun, M.; Chung-May, W.; J. Agric. Food Chem. 1996, 44, 1096.

11. Ahmad, A.; Misra, L. N.; Gupta, M. M.; J. Nat. Prod. 1993, 56, 456.

12. Moura, N. F.; Strapazzon, J. O.; Loro, F.; Morel, A. F.; Flach, A.; J. Essent. Oil Res. 2006, 18, 627.

13. Gonzaga, W. A.; Weber, A. D.; Giacomelli, S. R.; Simionatto, E.; Dalcol, I. I.; Dessoy, E. C. M.; Morel, A. F.; Planta Med. 2003, 69, 773.

14. Brophy, J. J.; Goldsack, R. J.; Forster, P. I.; Hutton, I.; J. Essent. Oil Res. 2000, 12, 285.

15. Simionatto, E.; Porto, C.; Dalcol, I. I.; da Silva, U. F.; Morel, A. F.; Planta Med. 2005, 71, 759 .

16. Ngassoum, M. B.; Essia-Ngang, J. J.; Tatsadjieu, L. N.; Jirovetz, L.; Buchbauer, G.; Adjoudji, O.; Fitoterapia 2003, 74, 284.

17. Menut, C.; Lamaty, G.; Bessiere, J. M.; Molangui, T.; Ayedoun, M. A.; Sossou, P. V.; Sohounhloue, K. D.; Djossou, L.; Houenon, J. G.; J. Essent. Oil Res. 2000, 12, 33.

18. Facundo, V. A.; Rezende, C. M.; Pinto, A. C.; de Morais, S. M.; J. Essent. Oil Res. 2003, 15, 402.

19. Trongtokit, Y.; Rongsriyam, Y.; Komalamisra, N.; Apiwathnasorn, C.; Phytother. Res. 2005, 19, 303.

20. Bowers, W. S.; Ortego, F.; Xiaoqing, Y.; Evans, P. H.; J. Nat. Prod. 1993, $56,935$.

21. Kamsuk, K.; Choochote, W.; Chaithong, U.; Jitpakdi, A.; Tippawangkosol, P.; Riyong, D.; Pitasawat, B.; Parasitol. Res. 2007, 100, 339.

22. Lu, X.; Zhang, Y.; Zhao, K.; Lu, Y.; Liu, Y.; Zhongguo Liangyou Xuebao 1996, 11,12 .

23. Marr, K. L.; Tang, C. S.; Biochem. Syst. Ecol. 1992, 20, 209.

24. Weenen, H.; Nkunya, M. H. H.; Bray, D. H.; Mwasumbi, L. B.; Kinabo, L. S.; Kilimali, V. A. E. B.; Wijnberg, J. B. P. A.; Planta Med. 1990, 56, 371.

25. Navarrete. A.; Hong, E.; Planta Med. 1996, 62, 250.

26. Sukari, M.; Aspollah, R.; Rahmani, M.; Choo, N. G. B.; Haron, M. D. J.; Ali, J. H.; Ahmad, F. B. H.; Orient. J. Chem. 1996, 12, 135.

27. de Moura, N. F.; Giancomelli, S. R.; Machado, E. C. S.; Morel, A. F.; Silveira, S. F. S.; Bittencourt, C. F.; Fitoterapia 1996, 69, 271.

28. http://flora.cria.org.br, acessada em Julho 2008.

29. Melo, M. F. F.; Zickel, C. S.; Acta Bot. Bras. 2004, 18, 73.

30. Facundo, V. A.; Morais, S. M.; Machado, M. I. L.; Matos, F. J. A.; Frota, L. C. M.; J. Essent. Oil Res. 1999, 11, 426.

31. Petrakis V. P.; Roussis, V.; Papadimitriou, D.; Vagias, C.; Tsitsimpikou, C.; Behavioural Processes 2005, 69, 303.

32. Bellakhdar, J.; Idrissi, A. I.; Canigueral, S.; Iglesias, J.; Vila, R.; J. Essent. Oil Res. 1994, 6, 523.

33. Santos-Gomes, P. C.; Fernandes-Ferreira, M.; Vicente, A. M. S.; J. Essent. Oil Res. 2005, 17, 73.

34. Zygadlo, J. A.; Lamarque, A. L.; Maestri, D. M.; Guzmán, C. A.; Lucini, E. I.; Grosso, N. R.; Ariza-Espinar, L.; J. Essent. Oil Res. 1994, 6, 407.

35. Adams, R. P.; Identification of Essential Oil Components by Gas Chromatography/Quadrupole Mass Spectroscopy, Allured Publishing Corporation: Illinois, 2001. 